\title{
Analytical techniques for steroid estrogens in water samples - A review
}

\begin{abstract}
In recent years, environmental concerns over ultra-trace levels of steroid estrogens concentrations in water samples have increased because of their adverse effects on human and animal life. Special attention to the analytical techniques used to quantify steroid estrogens in water samples is therefore increasingly important. The objective of this review was to present an overview of both instrumental and non-instrumental analytical techniques available for the determination of steroid estrogens in water samples, evidencing their respective potential advantages and limitations using the Need, Approach, Benefit, and Competition (NABC) approach. The analytical techniques highlighted in this review were instrumental and non-instrumental analytical techniques namely gas chromatography mass spectrometry (GC-MS), liquid chromatography mass spectrometry (LC-MS), enzyme-linked immuno sorbent assay (ELISA), radio immuno assay (RIA), yeast estrogen screen (YES) assay, and human breast cancer cell line proliferation (E-screen) assay. The complexity of water samples and their low estrogenic concentrations necessitates the use of highly sensitive instrumental analytical techniques (GC-MS and LC-MS) and non-instrumental analytical techniques (ELISA, RIA, YES assay and E-screen assay) to quantify steroid estrogens. Both instrumental and non-instrumental analytical techniques have their own advantages and limitations. However, the non-instrumental ELISA analytical techniques, thanks to its lower detection limit and simplicity, its rapidity and cost-effectiveness, currently appears to be the most reliable for determining steroid estrogens in water samples.
\end{abstract}

Keyword: Analytical technique; Instrumental; Non-instrumental; Steroid estrogens; Water samples 